\title{
ÜBERSICHTEN.
}

\section{ZUR ANALYSE DER GENITALEN BLUTUNGEN BEI NICHTSCHWANGEREN FRAUEN.}

\author{
Von \\ Prof. R. SCHRÖDER, Rostock.
}

„Unregelmäßige Blutungen, Fluor und Regelschmerzen sind die klinischen Zeichen der chronischen Endometritis." Diese Formel hat viele Jahre hindurch die Vorstellungen der Ärzte bei der Behandlung gynäkologisch kranker Frauen geleitet. Die chronische Endometritis zeigte bestimmite anatomische Charaktere und dokumentierte sich durch sie als eine offenbare Wucherung; nichts lag näher, als da. 3 man durch Entfernung der Wucherung auch die ihr zur Last gelegten klinischen Zeichen zu beseitigen glaubte. Der Erfolg gab vielfach diesen Gedankengängen offenbar recht, aber viele Mißerfolge haben doch dauernden $Z$ weifel wachgehaiten.

Wie ich im Heft 9 dieser Wochenschrift auseinandersetzte, ist die Auffassung der chronischen Endometritis als eines langsam entstandenen stationären Gebildes, in dessen Bereich die menstruelle Welle nur durch-Capillardilatation und subepitheliale Hämorrhagie periodisch zum Ausdruck kam, nicht richtig; ihre Bilder lassen sich vielmehr als bestimmte, im Fluß begriffene Phasen des alle 4 Wochen ablaufenden mensuellen Cyklus nachweisen. Die Einzelheiten dieses Ablaufes sind, soweit sie allgemeines Interesse beanspruchen, in jenem ersten Artikel dargestellt, auch deren biologische Bedeutung beleuchtet. Die Wichtigkeit dieser Kenntnisse für die Pathologie des Genitalapparates und für die so häufigen, alltäglich vorkommenden Krankheitsbilder will ich heute durch eine kurze zusammenfassende Besprechung der hierher gehörigen Anomalien beleuchten. Da die anatomische Basis jenes Krankheitsbildes der chronischen Endometritis sich als falsch gedeutet erwies, so fiel auch die theoretische Grundlage zum Verständnis jener eingangs erwähnten Zeichen in sich zusammen, neue Wege mußten gefunden werden.

Zunächst soll festgestellt werden, da $\beta$ die Symptomentrias der unregelmäBigen Blutungen, des Fluors und der Regelschmerzen sich keinesfalls als a priori zusammengehörig erweist; jedes dieser Symptome muß vielmehr für sich studiert und beurteilt werden. Tatsächtlich zeigt sich, daß in der Pathogenese der drei Symptome nur äußerst lockere Zusammenhänge auffindbar sind. So ist der Fluor in seiner Entstehung durchaus nicht einheitlich, Vulva, Vagina und Cervicalkanal können ihn erzeugen. Näheres kann in diesem Rahmen nicht darüber gesagt werden, ohne weiter ausgreifen zu müssen und vollständig abzuschweifen. Aber betont werden muß, daß das Endometrium corporis nur unter der Voraussetzung einer schweren eitrigen Entzündung an seiner Bildung beteiligt ist, abgesehen natürlich von Carcinomen, oberflächlich entzündeten Polypen oder Myomen usw.; sonst wird im Endometrium corporis kein Schleim gebildet, auch nicht während der Sekretionsphase, deren Sekretionsprodukte höchstens schleimähnliche, aber wäßrige Flüssigkeiten sind, die als Nahrung für das zu erwartende befruchtete Ei dienen sollen.

Die Regelschmerzen sind irgendwie in Zusammenhang zu denken mit den Muskelkontraktionen des menstruierenden Uterus, den wir ja in jenem Artikel mit dem abortierenden und gar gebärenden Uterus in Analogie setzen mußten; entweder vermehrte Widerstände für den Sekretabfluß, Rigidität der Weichteile, Hypotonien der Muskulatur oder entzündliche Prozesse der Umgebung, evtl. auch erhöhte psychische Perzeption normaler Bewegungen und Gefühlseindrücke können ätiologisch für den Schmerz in Frage kommen.

Am kompliziertesten gestaltet sich das Verständnis für die unregelmäßigen Blutungen. Es ist unmöglich, in einem räumlich begrenzten Artikel nur einigermaßen erschöpfend das Gebiet zu besprechen; auch können hier nur die Hauptgedankengänge die Richtung angeben, in der das Verständnis für den einzelnen Fall gesucht werden muß. Die Grundlage geben die normalen Vorgänge, deren große Widerstandsfähigkeit gegen die verschiedenartigsten Einwirkungen sich immer wieder nachweisen läßt, z, B. gegen die Einwirkungen der meisten gynäkologischen Affektionen: (Myome; Carcinome, Ovarialtumoren, Lageanomalien): Also das Ei mit seiner Follikel- und Corpusluteum-Bildung ist der treibende Faktor; das Tempo seiner Reifung und die Zeit seines Reifseins resp. der Termin seines Todes bestimmt das Tempo des Cyklusablaufes und damit auch das Tempo der Menstruationswiederkehr. In Abhängigkeit von der Wirkung des Eies und seines Follikels resp. seines Corp. 1ut. wandelt sich das wesentlichste Erfolgsorgan, das Endometrium corporis cyclisch um, bildet durch Proliferation eine dicke Funktionsschicht, kommt in Sekretion und wird durch Desquamation seiner ganzen funktionellen Schicht zu einer Wundfläche, aus der es blutet; die Epithelialisierung dieser Wundfläche leitet unter Wirkung eines neuen reifenden Eies in die neue Funktionalisproliferation über. Die Stärke derr Blutung ist abhängig, wie beim Abort und Partus, von der Menge des zuströmenden Blutes und von der Kontraktionskraft der Uterusmuskulatur. Für das Verständnis der von der Norm abweichenden Regelblutungen kommt es nun darauf an, diese eben angegebenen Einzelfaktoren zu analysieren und ätiologisch zu erforschen. Vor allem also ist das ätiologische Moment, das die Stärke und Dauer der einzelnen Regelblutung bedingt, in seinem Angriffspunkt an die Gefäßbahn und die Uterusmuskulatur gebunden, dasjenige jedoch, das Unregelmäßigkeiten in der Wiederkehr der Regelblutung herbeiführte, wirkt am Eiparenchym ein. Bei diesen grundsätzlichen Verschiedenheiten der Angriffspunkte ist es natüriich wesentlich; bei der Analyse des einzelnen Krankheitsbildes ebenfalls die Stärke und Dauer der Regelblutung einerseits und das Tempo der Wiederkehr andererseits anamnestisch genau festzulegen.

Die Ursachen abnormer Blutungsstärke lassen sich nach dem Gesagten in verschiedene Einzelrubriken sondern. Die Uterusmuskulatur kann kontraktionsschwach werden durch starke Abnutzung infolge zahlreicher Partus, infolge asthenischer Konstitution, bei Lageanomalien, bei Durchsetzung des Myometriums mit Myomknoten, infolge von Entzündungsprozessen in der Umgebung. Die Blutmenge, die zur Zeit der letzten Cyklustage zum Becken hinströmt, ist im Grunde abhängig von den prägraviden Reizen, die vom Reifei in letzter. Linie ausgehen, kann aber verstärkt werden durch abnorme sexuelle Reizungen im Sinne aktiver Hyperämie, ebenso durch entzündliche Prozesse im Becken, aber auch durch passive Hyperämie verschiedenster Art.

Die Ursachen abnormer Regelwiederkehr greifen, wie gesagt, im Eiparenchym selbst an. Schwache schädigende Reize lassen die Eizelle wohl reif werden und zur Ovulation kommen, aber bringen sie bald nach der Aisstoßung zum Absterben, daher kurze Corpus luteum-Dauer, kurze Seliretionsphase und rasch folgende Regelblutung infolge verfrühter Desquamatio mucosae. Stärkere Schädigungen beeinträchtigen, auch das Reifungstempo, dadurch Hinausschieben "der. nächsten Regel。 seltene Regelfolge; starke Beeinträchtigung; des Eiparenchyms führt zum Ausbleiben der Reifung und 
evtl. gar des Wachsens von Follikeln und Eiern (Amenorrhöe). Die Art der hier wirksamen Reize sind gegeben in allgemeinen Infektionskrankheiten, Tuberkulose, Stoffwechselañomalien, Herzdekompensationen, Wunderkrankungen, Ernährungsstörungen, endokrinen Krankheitsbildern, psychischen Faktoren usw.; aber auch primäre konstitutionelle Minderwertigkeiten können so zutage treten.

Das einzelne Krankheitsbild ist fast nie eindeutig, sondern mehrere Faktoren spielen eine Rolle; zu ihrer Erkennung ist eine genaue Untersuchung des ganzen Körpers dringend wichtig. Mit der Behebung der ursächlichen Faktoren verschwinden die Symptome; denn das ist die Quintessenz: Anomalien der cyclischen Blutungen sind stets sekundäre Zeichen eines übergeordneten Krankheitsbildes. Dieser Satz ist von größter Wichtigkeit für das therapeutische Vorgehen.

Im Gegensatz zu diesen cyclischen Blutungsanomalien (gewöhnlich mit Menorrhagien bezeichnet) stehen die unperiodischen, nichtcyclischen Genitalblutungen (gewöhnlich Metrorrhagien genannt). Sie können auftreten bei erhaltenem Regelzyklus und bei völliger Intaktheit seines anatomischen Ablaufes, ihn auch einmal völlig überdecken; dann kommt die abnorme Blutung z. B. durch submuköse Myome, Polypen, Carcinome, Erosionen oder auch per diapedesin bei Herzkrankheiten zustande. Andere funktionelle Blutungen bei anatomisch normalem Zylklus kommen in der prägraviden Phase (abnorme Durchlässigkeit der Capillaren) oder auch um die Ovulationszeit vor. In weiteren Fällen ist ein Regelzyklus anamnestisch und anatomisch nicht mehr erkennbar; sondern mehr oder weniger kurz nach einer Regel, die evtl. schon verstärkt war; treten unregelmäßig starke Blutungen von unregelmäßiger, oft durch Pausen unterbrochener. Dauer auf, gehen aber später in eine entweder regelmäßige oder auch verstärkte und verfrühte, oder wieder cyclische Regel über. Hier findet sich ätiologisch oft eine echte Endometritis, deren Infektionserreger kurz vor oder während der letzten Regel vom Halskanal her ascendiert waren und nach der letzten Desquamatio mucosae die übrigbleibende Basaliswunde derartig geschädigt hatten, daß eine neue Funktionalis sich trotz wirksamer Hormonreize vom Ovarium aus nicht hat bilden können. Quelle der Blutung ist hier die niedrige, schwer entzündete und hyperämische Korpusschleimhaut selber.

Zur Zeit der beginnenden Klimax, aber auch zur Menarchezeit kommen dann noch regel- ùnd cycluslose Dauerblutungen vor, die in ihrer Art durchaus dem Blutungsbild eines inkompletten Abortes ähneln können, häufig eine rasch fortschreitende sekundäre, aber auch aplastische Anämie erzeugen und die Kranken schwer schädigen. In diesen Fällen. findet man dann das Bild, das schon früher als Endometritis fungosa bekannt war: unregelmäßig stark gewucherte, oft cystische Drüsen in ungleich dichtem Stroma, dessen Gefäße leichte Dilatationen zeigen, thrombosieren und dadurch allmählich um sich greifende und fortschreitende nekrobiotische Prozesse in der abnormen Schleimhaut bedingen. Die Quelle der Blutung sind diese thrombotischen und nekrotisierenden Bezirke der Schleimhaut selbst. Die Ursache der ganzen Wucherung aber ist in Abnormitäten der Ei- und Follikelreifung gegeben. In den Ovarien solcher Fälle findet man nicht, wie sonst bei cyclischen Regelblutungen, den zum Zyklus gehörigen jeweiligen Follikel resp. das Corp. lut., und das in entsprechender Rückbildung begriffene Corp. 1ut. des vorhergehenden Zyklus, ja noch das des weiter zurückliegenden, mit anderen Worten, man findet nicht die verschiedenen Corpus 1ut.-Generationen, die auf die abgelaufenen Zyklen hindeuten, sondern hier fehlen die früheren Generationen von Corpora lutea bis auf vielleicht sehr weit zurückliegende Formationen, und es finden sich nur große reifende oder reife Follikel mit Eizelle und dicker, fast hypertrophischer Membrana granulosa; sie können entweder einzeln oder $\mathrm{zu}$ mehreren vorkommen. Sie sind die eigentlichen treibenden Faktoren dieser Krankheitsbilder (Metropathia haemorrhagica in engerem Sinne). Die Follikel sind reif geworden, aber haben das $\mathrm{Ei}$ nicht freigegeben; gingen sie durch Atresie vorzeitig zugrunde, so folgten gleich neue, reifende Follikel, so daß die Wirkung des reifenden Follikels persistent $\mathrm{blieb}$. Diese ist die gesteigerte Proliferationswirkung auf die Funktionalis, ähnlich der, wie sie in der ersten Hälfte des normalen Zyklus in normaler Stärke auftritt. Das Abnorme der über das gewöhnliche Maß hinausgetriebenen Proliferation spricht sich in der UnregelmäBigkeit, in der Dicke der Schleimhaut, den abnormen Drüsenformen und der Unmöglichkeit, eine einheitliche, über die ganze Korpusschleimhaut gleichmäßig ausgebildete Phase erkennen zu können, schließlich aus dem Fehlen typischer Sekretionsbezirke aus. Die Röntgenkastration ist in solchen Fällen die Therapie der Wahl, da der treibende Faktor, der abnorme Follikel, sicher durch sie zerstört wird; in der Klimaxzeit ist sie zweifellos das einzig Richtige; in den Fällen der Pubertätszeit muß man vorerst alle anderen auf den Follikelapparat schädigend einwirkenden, allerdings unsicheren Mittel durchprobieren (Adrenalin, Hypophysenpräparate, Corp. lut.-Trockenpräparate (?) usw.).

Wie hier ein persistierender Follikel Regelwidrigkeiten bedingt, soll auch ein Corpus luteum persistens die fällige Regelblutung hinauszögern und nach seiner Rückbildung eine abnorme Blutung, dann eine echte verstärkte Regel bedingen; vielleicht ist hier aber ein versteckter Abort im Spiel, also eine Krankheitsursache, die ebenso wie eine extrauterine Gravidität nicht in diese Besprechung gehört.

Die unperiodischen, nichtcyclischen Blutungen haben nach dem Gesagten inre Ursache in abnormen Formationen im Uterus, selbst auch die Follikelpersistenz hat die abnormen Blutungen lediglich durch Vermittlung der abnormen Uterusschleimhaut zur Folge. Das setzt diese sog. "Metrorrhagien" in scharfen Gegensatz zu jenen, lediglich als Sekundärsymptome aufzufassenden "Menorrhagien". Mehr denn je muB deshalb zur Analyse von Blutungen auf die genaue Differenzierung des anamnestischen Regelbildes geachtet werden, das zusammen mit genauer Untersuchung des Genitale und des gesamten übrigen Körpers die meisten Fälle von Regelanomalien dem Verständnis des Arztes so nahe bringt, daß er die geeignete Therapie einschlagen kann.

\section{ORIGINALIEN.}

\section{ÜBER DIE AUTOTOXISCHE INTRAGLOBULÄRE SULFHÄMOGLOBINÄMIE.}

\author{
Von
}

Prof. A. A. Hijmans van den Bergh und Dr. H. Engelkes. Aus der medizin, Klinik zu Utrecht.

Die autotoxische Sulfhämoglobinämie beim Menschen wurde zuerst im Jahre 1905 beschrieben ${ }^{1}$ ). Zwar hatten früher schon Emminghaus, BETZ, SENATOR ${ }^{2}$ ) merkwürdige

3) EIJMANS V. D. BERGH, Dtsch. Arch. f. klin. Med. 83, S. 86. Ig05, HIJMANS V. D. BERGH u. GRUTTERINK, Berl, klin. Wochenschr. Iga6, I.

2) Berl, klin. Wochenschr, I872, IX, 477 und 49r. Beri. klin. Woohenschr. I868, 2) Berl,
Fälle von sog. Hydrothionämie beobachtet, welche mit mehr oder weniger großer Wahrscheinlichkeit als Schwefelwasserstoffvergiftung aufgefaßt wurden. Es waren aber unklare Krankheitsbilder, und jedenfalls fehlte der Nachweis des Sulfhämoglobinspektrums, welches für die von uns studierten Fälle gerade charakteristisch ist. Die Beobachtung der letztgenannten Krankheitsfälle fußte auf den wichtigen Veröffentlichungen von STotrvis ${ }^{1}$ ) und von TALMA $^{2}$ ) über die autotoxische enterogene Cyanose. Diese beiden Autoren beschrieben im Jahre 1902 fast gleichzeitig und unabhängig

${ }^{2}$ ) STOKVIS, Nederlandsch Tijdschr. v. Geneesk. 2, 678. rgoz; und Festschr. f. v. LEYDEN,

2) TALMA, Nederlandsch Tijdschr. v. Geneesk. 2, 72x. I902, 